MS32-P20

\section{Molecular and crystal structure analysis of two new Oxazol-5-One derivatives}

Merve İzmirli' ${ }^{1}$, İbrahim Hanif Nazlı ${ }^{1}$, Derya Topkaya Taşkıran², Serap Alp ${ }^{2}$, Muhittin Aygün ${ }^{3}$

1. Graduate School of Natural and Applied Science, Dokuz Eylül University, İzmir, Turkey

2. Chemistry, Dokuz Eylül University, İzmir, Turkey

3. Physics, Dokuz Eylül University, İzmir, Turkey

email: izmirlimerve@yahoo.com

Two new oxazole-5-one derivatives, known as unsaturated azlactones, were synthesised due to the optical and biochemical importance [1,2]. These fluorescent molecules which are the cyclic anhydrides of $\mathrm{N}$-acylaminoacids were formed by using Erlenmeyer azlactone reactions with aldehyde condensation. Molecular and crystal structures of 4-(N,N-diethylaminophenylmethylene)-2-(p-methoxyphenyl)oxazol-5-one (1) and 4-(N,N-diethylaminophenylmethylene)-2-(p-nitrophenyl)oxazol-5-one (2) have been determined by single crystal X-ray diffraction method. The data collections were carried out with Rigaku Oxford XCalibur diffractometer, cell refinements and data reductions were executed using CrysAlisPro program [3]. Using Olex2, structures were solved with the SHELXT structure solution program and refined by full-matrix least-squares against F2 using SHELXL refinement package. The crystal structure of the compound 1 is stabilized $\pi-\pi, \mathrm{C}-\mathrm{H} \ldots \pi$ and $\mathrm{C}-$ H...O type weak interactions, while the crystal structure of the compound 2 is stabilized intermolecular $\pi-\pi, N-$ $\mathrm{O} \ldots \pi$ and $\mathrm{C}-\mathrm{H} . . \mathrm{O}$ type weak interactions. There are also intramolecular $\mathrm{C}-\mathrm{H}$... $\mathrm{N}$ type weak interactions for both molecules.
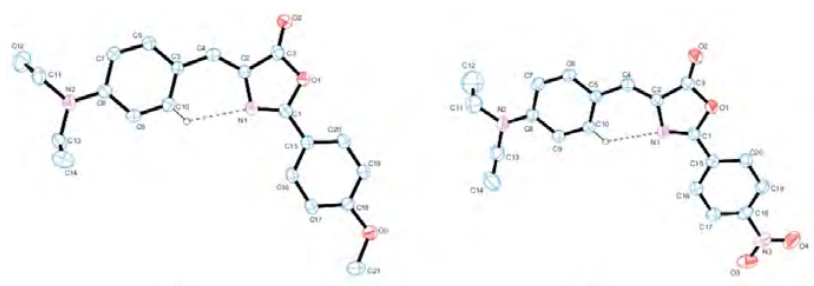

References:

[1] Öztürk. G. et al. (2008). Dyes Pigm. 76, 792-798.

[2] Khan. K. M. et al. (2008). Lett. Drug Des. Discovery. 5, 52-56.

[3] CrysAlisPro Software System, Version 1.171.38.43, Rigaku

Corporation, Oxford, UK, 2015.

Keywords: azlactone, X-ray crystallography, weak interactions
MS32-P21

\section{Molecules for electronics: Understanding their intramolecular properties towards their incorporation in devices}

Berta Gómez-Lor ${ }^{1}$, Angela Benito ${ }^{1}$, Constanza Ruiz ${ }^{1}$, Marcelo Echeverri $^{1}$

1. New Architectures in Materials Science, ICMM, CSIC, Madrid, Spain

email:bgl@icmm.csic.es

In recent years, the field of organic electronics has suffered enormous progresses. In fact, this area has evolved in only a few years from basic research to the development of devices such as organic light emitting diodes (OLEDs), organic photovoltaics (OPVs) and organic field effect transistor (OFETs) which are already reaching the market. The soft character of organic materials allows their solution processing and renders them very attractive in the development of flexible, light weight and low cost devices.

At the molecular level, fundamental parameters towards the incorporation of molecular materials in (opto)electronic devices -such as, light emission or absorption, energy levels... - can be designed "a la carte" thanks to the deep knowledge that has been acquired in the last few years through numerous structure-properties studies. However, properties of molecular materials are governed not only by those of individual molecules but also by the way they are spatially ordered. Single crystal analysis provides invaluable information on how molecules are arranged with respect to their surroundings and on the nature of intermolecular interactions among neighboring units in the bulk materials.

In this context we present here different examples of organic materials with light emitting and/or charge transport properties recently developed in our research group and show how a good understanding their intramolecular interactions (achieved through single crystal X-ray analysis) has allowed us to optimize their final properties.

Keywords: organic materials, charge transport, light emission 